\title{
Modeling the Thrombopoietic Effects of Burn
}

\author{
Jacqueline Wentz ${ }^{1,}{ }^{*}$, Darren Oldson ${ }^{1}$, Daniela Stricklin ${ }^{1}$
}

\begin{abstract}
Our group is currently developing physiologically based models to understand the impact of radiation combined injury and aid in casualty predictions for catastrophic scenarios. We have developed a model that describes the impact of burn on thrombopoiesis with the aim of integrating it into an existing radiation-effects model. Thrombocytopenia can be a life-threatening condition due to the role of platelets in hemostasis and the immune response. Thermal injury is known to cause thrombocytopenia as well as a subsequent thrombocytosis. Key biological effects of burn were identified, mathematically interpreted, and implemented. These effects include a decreased platelet lifespan and an increased repopulation rate due to increased levels of thrombopoietin within the system. The model predicts platelet and precursor cell concentrations over time, providing details on time, magnitude, and duration of thrombocytopenias. Parameter values were obtained through both the analysis of experimental data and the optimization of the model to observational data on platelet counts following burn. The model was subsequently validated against other datasets and simulations were performed to better understand the characteristics of the model. The model describes the effects of thermal injury on thrombopoiesis, providing realistic predictions that reflect the trends observed in platelet concentrations.
\end{abstract}

Keywords: thrombopoiesis, burns, mathematical model

\section{Introduction}

Many radiological incidents result in patients with multiple types of injuries. For example, $65-70 \%$ of people are expected to suffer from both radiation exposure and trauma or burn following a nuclear event. In the 1986 Chernobyl reactor incident, $10 \%$ of the victims received both significant radiation and burn injuries [25]. Our group is currently developing health effects models to better understand the impact of radiation combined injury and aid in casualty predictions for catastrophic scenarios. Since very little experimental data are available on combined injury, physiologically based mathematical modeling can aid in predicting the potentially synergistic mechanistic interactions in combined injury, thereby affording more accurate casualty assessments and predictions of the clinical course of the injuries. Using a model structure originally developed for describing the impact of radiation exposure on blood cell kinetics, we have developed model parameters that describe the impact of burn on thrombopoiesis. We will merge these parameters into the radiation effects model to better understand the impact of radiation and burn injury. In this paper, we present our model that describes thrombocyte kinetics after thermal injury which can help to enhance the mechanistic understanding of thermal injuries and eventually aid

\footnotetext{
${ }^{1}$ Applied Research Associates, Inc., Arlington, VA

${ }^{*}$ Correspondence: jwentz@ara.com
} 
in our understanding of the synergistic health effects of burn and radiation exposure on thrombopoiesis.

In 2012, approximately 450,000 people in the United States experienced burn injuries that required medical treatment, and 3,400 people died due to fire and burn [1]. While significant data has been collected on hematopoietic cell kinetics after burn, to our knowledge, no attempt to develop a hematopoietic model of burn injury has been made to date.

Burn is known to lead to states of thrombocytopenia and thrombocytosis. These states can be life-threatening due to platelets' role in hemostasis and the immune response. In pediatric burn patients a platelet count below $100 \cdot 10^{9} \mathrm{~L}^{-1}$ was a predictor of mortality [12]. Studies in adult populations found platelet count nadirs in burn patients were highly correlated with mortality $[20,35]$. Both thrombocytopenia and thrombocytosis at the time of hospitalization were predictors of mortality in patients with community-acquired pneumonia [22]. Thus, the development of a mechanistic model of thrombopoiesis following burn could help predict how burn and radiation combined injuries influence cell kinetics and understand the correlation with the time course of clinical symptoms and probability of mortality.

Several other mathematical models of thrombopoiesis have previously been developed, including models of radiation injury [32, 7], conventional chemotherapy [28], megakaryocyte growth and development factor; PEG-rHuMGDF [10], and thrombopoietin (TPO) [30]. However, to date, no model describing the effects of thermal injury on thrombopoiesis exists. Rather than starting from scratch, we chose to adapt a radiation-effects thrombopoiesis model developed by Smirnova et al. [16, 31, 32]. This particular model was chosen because the model was (1) relatively simple when compared with other existing models and (2) already designed to incorporate acute radiation exposure. Thus, simulating radiation combined injury would be the natural next step.

Using the percentage of total body surface area burned (\%TBSA) and the blood thrombocyte concentration under healthy conditions as inputs, the model predicts platelet and precursor cell concentrations over time. The model has been constructed such that the burninduced alterations in rates and the subsequent recovery times can be biologically justified using experimental data. After incorporating structural changes and extracting parameter values from empirical data, the model was both optimized and validated against serial data on platelet counts in burn patients. The model provides a means for calculating the time, magnitude and duration of thrombocytopenias as well as hypotheses for the biological mechanisms behind these results.

\section{Background}

Thrombopoiesis is initiated by hematopoietic stem cells (HSCs) in the bone marrow. HSCs have the ability to self-replicate as well as differentiate into progeny of various cell lineages, including the thrombopoietic cell lineage. The earliest identifiable thrombopoietic cell is the megakaryoblast which proceeds to differentiate into megakaryocytes. Megakaryocytes mature in the bone marrow through a series of endomitoses, gradually increasing levels of ploidy. Once fully mature, each megakaryocyte releases thousands of platelets into the blood stream.

There are a couple known feedback mechanisms that help humans maintain a healthy level of platelets within the blood. The principle mediator of thrombopoiesis is TPO; for a review of TPO regulation see [15]. TPO acts as a thrombopoietic stimulator, while platelets and megakaryocytes provide a negative feedback mechanism by receptor-mediated uptake and destruction of TPO. As levels of megakaryocytes and platelets within the system decrease, there is more TPO which leads to an increased stimulation of thrombopoietic progenitors. In a second feedback mechanism, the concentration of platelets within the blood directly affects megakaryocyte ploidy levels. As the platelet concentration decreases, the ploidy level of megakaryocytes increases and more platelets are produced. These systems 
allow for the body to respond if platelet levels are too high or too low.

Burn affects the thrombopoietic system through a couple known mechanisms. In both rodents and humans, platelet lifespan is known to decrease following burn [6, 29]. This is potentially caused by the consumption of platelets due to coagulation at the burn wound site and other locations [21]. Burn tissues release thromboplastin which leads to the formation of microthrombi at distant sites which are then removed by the reticuloendothelial system [36]. The mechanisms of increased platelet decay are known to be extrinsic [6], reinforcing the notion that factors in the serum cause the increased platelet consumption. The increased rate of decay and subsequent thrombopenia are dependent on the size of the burn [21]. As burn size increases, a more drastic decrease in platelet counts is observed.

The sustained quality of the thrombocytosis following burn implies there must be another mechanistic change in the model. A temporary thrombocytosis could be explained as a reaction to the thrombopenia caused by burn, however the sustained quality suggests an increased repopulation rate of mitotic precursor cells. In a study of burn patients which excluded patients with low platelet levels $\left(<100 \cdot 10^{9} \mathrm{~L}^{-1}\right)$, circulating levels of TPO increased [19]. Furthermore, the concentration of IL-6, a cytokine known to cause TPO transcription in the liver [14], increases in burn patients within the first few hours postburn [4, 5,27 and remains detectable through at least 5 weeks postburn [5]. The magnitude of increase in IL-6 levels has been correlated with burn size [27, 17], although one study did not observe this relationship [5]. IL-6 stimulates thrombopoiesis in vivo in mice and monkeys. Following a dosing regimen of IL-6 in mice, platelet counts increased significantly by day 4 in a dose-dependent fashion [13]. In monkeys, platelet counts increased significantly on day 7 of IL-6 treatment [2]. Taken together, this evidence suggests that burn leads to increased levels of IL-6, which in turn increases circulating TPO levels resulting in an increased HSC repopulation rate.

\section{Methods}

\subsection{Baseline Model Structure}

Models from Smirnova et al. 2011, 2012 were used as a starting point for establishing a thrombopoiesis model for burn [31, 32]. Smirnova's models incorporate radiation effects; however, when developing our burn-only model the effects of radiation were removed from the system and will not be presented here. Cells in the thrombopoietic lineage are divided into three functional compartments. The first compartment $\left(X_{1}\right)$ consists of mitotic cells in the bone marrow which includes progenitor cells ranging from hematopoietic stem cells to megakaryoblasts. The second compartment $\left(X_{2}\right)$ consists of the megakaryocytes in the bone marrow, and the third compartment $\left(X_{3}\right)$ consists of platelets in the bloodstream. The dynamics of this system are initially described using differential equations taken directly from Smirnova's original model [31], where $\dot{x}_{i}$ represents the change in concentration of cells in compartment $X_{i}$ over time $(i=1,2,3)$ :

$$
\begin{aligned}
& \dot{x}_{1}=B\left(x_{1}, x_{2}, x_{3}\right) x_{1}-\gamma x_{1}, \\
& \dot{x}_{2}=f\left(x_{3}\right) \gamma x_{1}-\delta x_{2}, \\
& \dot{x}_{3}=\sigma \delta x_{2}-\psi x_{3},
\end{aligned}
$$


where

$$
\begin{aligned}
B\left(x_{1}, x_{2}, x_{3}\right) & =\text { repopulation rate of the dividing progenitor cells, } \\
\gamma & =\text { transition rate from } X_{1} \text { to } X_{2}, \\
\delta & =\text { transition rate from } X_{2} \text { to } X_{3}, \\
\psi & =\text { rate of platelet decay, } \\
f\left(x_{3}\right) & =\text { feedback of } x_{3} \text { on ploidy levels, } \\
\sigma & =\text { number of platelets produced per megakaryocyte. }
\end{aligned}
$$

Although (1)-(3) were taken directly from Smirnova's model, the description of the mediator feedback effect on the $x_{1}$ repopulation rate $(B)$ has been changed from inhibitory to stimulatory as described in Section 3.1.1.

\subsubsection{Effects of stimulatory mediator}

The rate of $x_{1}$ repopulation $B$ is given by the following equation

$$
B=\frac{\alpha}{1+\frac{K}{I}}
$$

where

$$
\begin{aligned}
& \alpha=\text { maximum rate of } x_{1} \text { repopulation, } \\
& I=\text { generic stimulatory mediator, } \\
& K \text { determines the strength of the mediator effect. }
\end{aligned}
$$

When $I=K$, the $x_{1}$ repopulation rate is $\alpha / 2$, half the maximum value. In Smirnova's original model, the mediator $I$ has an inhibitory effect on the $x_{1}$ repopulation rate. The inhibitory mediator is created by all cells in the model. Thus, as the number of cells increases the $x_{1}$ repopulation rate decreases. Here, we have re-characterized this mediator with a stimulatory effect to provide a more accurate representation of TPO. Thus, in the new model, cell concentrations in compartments $X_{i}(i=1,2,3)$ affect the decay rate of the mediator: as the number of cells increases, the mediator decays at a faster rate. The change in mediator with time is defined as

$$
\frac{d I}{d t}=G-H\left(\theta_{1} x_{1}+\theta_{2} x_{2}+\theta_{3} x_{3}\right) I
$$

where

$$
\begin{aligned}
G & =\text { production rate of the mediator, } \\
H \theta_{i} & =\text { effect of } x_{i} \text { on the decay rate of the mediator. }
\end{aligned}
$$

Assuming $I$ reaches a steady state relatively rapidly (compared to the time scale over which the cell populations change), we can assume $d I / d t \approx 0$, solve for $I$, and insert the solution into (4):

$$
B\left(x_{1}, x_{2}, x_{3}\right)=\frac{\alpha}{1+\beta\left(\theta_{1} x_{1}+\theta_{2} x_{2}+\theta_{3} x_{3}\right)}
$$

where

$$
\beta=\frac{K H}{G}
$$




\subsubsection{Effects of ploidy}

The second feedback mechanism, taken directly from Smirnova, describes how $x_{3}$ concentration affects ploidy levels [31]. As the cell concentration in compartment $X_{3}$ increases, the megakaryocyte ploidy decreases. This effect on the megakaryocyte ploidy is incorporated into the model by multiplying the transition rate into the $x_{2}$ compartment by a ploidy factor $f$, where

$$
f\left(x_{3}\right)=\frac{1}{\lambda+(1-\lambda)\left(x_{3} / \bar{x}_{3}\right)},
$$

$\bar{x}_{3}$ is the concentration of cells in compartment $X_{3}$ under healthy conditions, and $\lambda$ is a fitted parameter that determines the strength of the ploidy feedback mechanisms ( $\lambda$ must take on a value between 0 and 1). If $\lambda=0$, the feedback strength is maximized, and if $\lambda=1$, the cell concentration in compartment $X_{3}$ has no effect on ploidy levels.

\subsubsection{Megakaryocyte maturation}

In both rodents and humans there is a delay between the time of burn and when the system enters a state of thrombocytosis. This delay can be explained as the necessary time for the burn-induced surge of thrombopoietic progenitor cells to travel through the mitotic and post-mitotic compartments. In Smirnova's model, the transit time through the post-mitotic $X_{2}$ compartment is exponentially distributed with an expected transit time $E\left[t_{M K}\right]$ of $1 / \delta$. We have adjusted the megakaryocyte maturation model with the aim of preserving the expected transit time $E\left[t_{M K}\right]$ while incorporating a more realistic transit time distribution. Specifically, we have changed the outflow from compartment $X_{2}$ from $\delta x_{2}$ to $F$, where $F$ is a function of the inflow history $h(t)=f \gamma x_{1}(t)$ :

$$
F(t)=\int_{-\infty}^{t} h(\tau) g(t-\tau ; n, n \delta) d \tau
$$

where $n \in \mathbb{N}$ and $g(x ; a, b)=b^{a} \frac{1}{\Gamma(a)} x^{a-1} e^{-b x}$ is the density of the gamma distribution (expectation $a / b$, variance $\left.a / b^{2}\right) \cdot t_{M K}$ is therefore gamma distributed, $E\left[t_{M K}\right]=1 / \delta$, and $\operatorname{Var}\left[t_{M K}\right]=n /(n \delta)^{2}=E^{2}\left[t_{M K}\right] / n$. As $n$ increases, the transit time variance decreases; thus, cells that enter the $X_{2}$ compartment are less likely to leave immediately. Using this modification to (1)-(3) and the Linear Chain Trick, the model can be written as

$$
\begin{aligned}
\dot{x}_{1} & =B\left(x_{1}, x_{2}, x_{3}\right) x_{1}-\gamma x_{1}, \\
\dot{x}_{2,1} & =f\left(x_{3}\right) \gamma x_{1}-n \delta x_{2,1}, \\
\dot{x}_{2, i} & =n \delta x_{2, i-1}-n \delta x_{2, i} \quad(i=2,3, \ldots, n), \\
\dot{x}_{3} & =\sigma n \delta x_{2, n}-\psi x_{3}, \\
x_{2} & =\sum_{i=1}^{n} x_{2, i} .
\end{aligned}
$$

\subsection{Developing parameters for the impact of burn}

\subsubsection{Platelet lifespan}

In the baseline model, platelet lifespans are assumed to be exponentially distributed with an expected value of $T_{p l t}$ or $1 / \psi$. To model an increased rate of platelet decay, $\psi$ becomes a function of burn size fraction $S$ and time postburn $t: \psi=\psi(S, t)$. Thus, two dependencies must be determined; namely, that of $\psi$ on burn size and time postburn.

To determine the dependence of platelet decay on burn wound size, data on platelet lifespan changes in humans as a function of burn size were fit to exponential, linear, and Hill decay (Hill coefficient=1) functions (Table 1). The functions implemented all contained two parameters, and thus the residual sum-of-squares was used to determine which function 

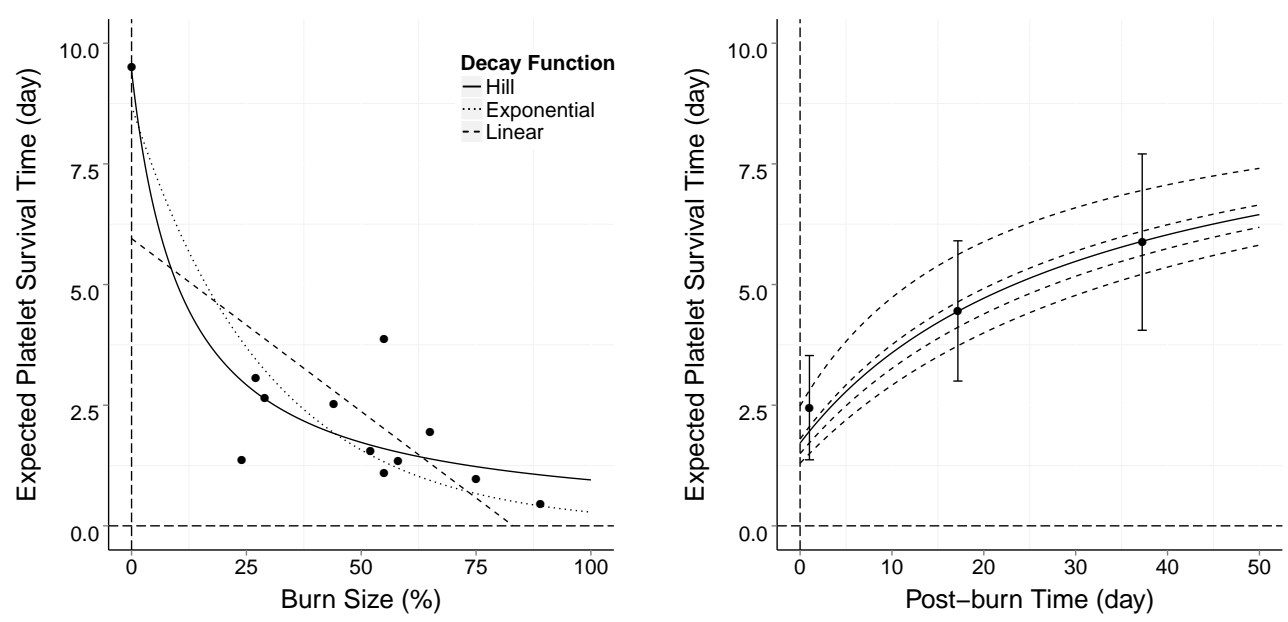

Figure 1: Comparison of platelet survival time following burn with model simulations. Left: Relationship between burn wound size at approximately 0.5 days postburn and expected platelet survival time. Data points as reported in [29] represent the calculated platelet survival times for individuals with varying burn wound sizes. Three optimized decay models are shown. Right: Relationship between the postburn time and the expected platelet survival time. Data points as reported in [29] represent mean $\pm \mathrm{SE}$ of the expected platelet survival times of 5 burn subjects at various times postburn. The solid line represents the simulation of the mean expected platelet survival time (19) and the dashed lines represent the simulation of the individual platelet survival times as a function of time postburn (17).

best described the data. Figure 1 (left) shows the three optimized models overlaid on the experimental data [29]. The following Hill function best described the data:

$$
T_{p l t}(S)=\frac{1}{\psi_{0}} \cdot \frac{1}{1+\frac{S}{d}}
$$

where $d$ determines the burn fraction at which $T_{p l t}$ is half the normal value or $1 / 2 \psi_{0}$.

Next, time dependence was added to this equation. Assuming the rate of recovery is not dependent on \%TBSA, this dependence can be incorporated by making the parameter $d$ time-dependent:

$$
T_{p l t}(t, S)=\frac{1}{\psi_{0}} \cdot \frac{1}{1+\frac{S}{d(t)}} .
$$

Assuming $T_{p l t}$ gradually returns to normal levels and that the change in decay is maximized immediately following burn, the function $d(t)$ should be defined for $t \geq 0$ such that at $t=0$, $d(t)$ is minimized and $\lim _{t \rightarrow \infty} d(t)=\infty$. The following linear function satisfies this criteria:

$$
d(t)=d_{0}+d_{1} t .
$$

Thus, the model of platelet lifespan is

$$
T_{p l t}(t, S)=\frac{1}{\psi_{0}} \cdot \frac{1}{1+\frac{S}{d_{0}+d_{1} t}} .
$$

Based on the data reported in [29] describing the relationship between \%TBSA and platelet lifespan shown in Figure 1 (left), a value for $d(t)$ is calculated and thus $d_{0}$ is written as a function of $d_{1}$. The data was obtained from subjects between 0 and 1 day postburn; we 
Table 1: Results of fitting data on platelet lifespan to linear, exponential, and Hill decay functions.

\begin{tabular}{ccccccc} 
Decay Function & Formula & $\mathrm{c}$ & Std. Error & $\mathrm{d}$ & Std. Error & rss \\
\hline Linear & $c-S / d$ & 5.95 & 1.13 & 0.140 & 0.041 & 29.58 \\
Exponential & $c e^{-S / d}$ & 8.71 & 1.19 & 0.292 & 0.053 & 15.24 \\
Hill & $\frac{c}{1+d S}$ & 9.43 & 0.97 & 0.112 & 0.027 & 9.387
\end{tabular}

will simplify this by using the midpoint and assuming all the data was obtained at $0.5 \mathrm{~d}$ postburn. Thus, as given in Table 1 , the optimized value for $d(t=0.5)$ is $0.112 \pm 0.027$ and

$$
d(t=0.5)=d_{0}+0.5 d_{1}=0.112 \Rightarrow d_{0}=0.112-0.5 d_{1} .
$$

To find the value for $d_{1}$, temporal data from human studies on platelet lifespans at different time points following burn were used. A serial study was performed on the platelet lifespan of 5 patients and provides the mean expected platelet lifespan for the patient group at various times postburn [29].

The mean platelet lifespan for $n$ subjects, each with a specified burn size, at time $t$ is given by the following formula:

$$
\bar{T}_{p l t}(t)=\frac{1}{n \psi_{0}} \sum_{i=1}^{n} \frac{1}{1+\frac{S_{i}}{d_{0}+d_{1} t}}=\frac{1}{n \psi_{0}} \sum_{i=1}^{n} \frac{1}{1+\frac{S_{i}}{\left(0.112-0.5 d_{1}\right)+d_{1} t}}
$$

where $S_{i}$ is the fraction of surface area burned for subject $i=1,2, \ldots, n$. This equation was fit to the experimental data on 5 patients by setting $n=5$ and $S_{i}=\% \mathrm{TBSA} / 100 \%$ for subject $i$. Figure 1 (right) shows the optimized model output overlaid on the data, and the expected individual results using (17). The optimized value of $d_{1}$ is $0.0185 \mathrm{~d}^{-1}$ making $d_{0}=0.103$.

To determine the rate of platelet decay, assuming the platelet lifespans are exponentially distributed at all time points following burn, we calculate $\psi(t, S)=1 / T_{p l t}(t, S)$ :

$$
\psi(t)=\psi_{0}\left(1+\frac{S}{d_{0}+d_{1} t}\right) .
$$

Thus, at a specified time point following burn, the rate of platelet decay is linearly related to the \%TBSA.

\subsection{Mediator levels}

To model the effect of burn on TPO production through IL-6, the rate of mediator production $G$ is modified such that it increases immediately after burn and gradually returns to normal. The following equation describes how mediator generation changes with time:

$$
\frac{d I}{d t}=G\left(1+\frac{f_{0}(S)}{1+a_{0} t}\right)-H\left(\theta_{1} x_{1}+\theta_{2} x_{2}+\theta_{3} x_{3}\right) I
$$

where $f_{0}(S)$ is the maximum relative increase in the rate of production, and $a_{0}$ defines how quickly values return to control levels. Assuming again that $d I / d t \approx 0$, the rate of $x_{1}$ repopulation becomes

$$
B(t)=\frac{\alpha}{1+\frac{H K}{G}\left(\theta_{1} x_{1}+\theta_{2} x_{2}+\theta_{3} x_{3}\right) /\left(1+\frac{f_{0}(S)}{1+a_{0} t}\right)} .
$$

The function $f_{0}(S)$ is unknown but is assumed to be related to $S$ such that $f_{0}(S=0)=0$. The function is defined as $f_{0}(S)=b_{0} S^{1 / c_{0}}$ where $b_{0}$ and $c_{0}$ must be greater than 0 . Thus 
the final equation for $B(t)$ is

$$
B(t)=\frac{\alpha}{1+\frac{H K}{G}\left(\theta_{1} x_{1}+\theta_{2} x_{2}+\theta_{3} x_{3}\right) /\left(1+\frac{b_{0} S^{1 / c_{0}}}{1+a_{0} t}\right)} .
$$

Values for $b_{0}, a_{0}$, and $c_{0}$ were found through model optimization.

\subsection{Finalized model equations}

The structure of the thrombopoiesis model, incorporating the effects of burn, are as follows:

$$
\begin{aligned}
\dot{x}_{1} & =B(t) x_{1}-\gamma x_{1}, \\
\dot{x}_{2,1} & =f \gamma x_{1}-n \delta x_{2,1}, \\
\dot{x}_{2, i} & =n \delta x_{2, i-1}-n \delta x_{2, i} \quad(i=2,3, \ldots, n), \\
\dot{x}_{3} & =\sigma n \delta x_{2, n}-\psi(t) x_{3}, \\
x_{2} & =\sum_{i=1}^{n} x_{2, i},
\end{aligned}
$$

where

$$
\begin{aligned}
B(t) & =\frac{\alpha}{1+\beta\left(\theta_{1} x_{1}+\theta_{2} x_{2}+\theta_{3} x_{3}\right) /\left(1+\frac{b_{0} S^{1 / c_{0}}}{1+a_{0} t}\right)}, \\
\psi(t) & =\psi_{0}\left(1+\frac{S}{d_{0}+d_{1} t}\right) \\
f & =\frac{1}{\lambda+(1-\lambda)\left(x_{3} / \bar{x}_{3}\right)} .
\end{aligned}
$$

\subsection{Parameter estimation}

For the baseline model without burn effects, parameters were taken directly from Smirnova's 2012 human thrombopoiesis model. Exceptions to this are as follows:

- The rate of platelet decay $\psi$ in Smirnova's thrombopoiesis model is $0.2 \mathrm{~d}^{-1}$ which gives an expected platelet lifespan of 5 days. However, the generally accepted platelet lifespan in humans is 9-10 days [29]. Therefore, in our model we have changed $\psi_{0}$ to $0.11 \mathrm{~d}^{-1}$.

- Incorporating an $x_{2}$ transit time that follows a gamma distribution involved adding a shape parameter $n$; equivalently $n$ defines the number of $x_{2}$ subcompartments. The value of this parameter was set to 4 .

Using the equilibrium values of $x_{1}, x_{2}$, and $x_{3}$ as reference values, $\beta$ (29) does not appear in the nondimensional equations. The baseline model parameter values are given in Table 2. For the burn-dependent parameters, $d_{0}$ and $d_{1}$ were determined as described in Section 3.2.1 while $a_{0}, b_{0}$, and $c_{0}$ were determined through optimization, and their finalized values are given in Table 3.

\subsection{Optimization procedures}

To determine nonlinear fits to experimental data, nonlinear least squares (nls) from the stats package was performed in $\mathrm{R}$ [26]. All optimization routines were performed in $\mathrm{R}$ using functions from the FME package [33]. The function modCost was used to estimate the model output residuals by running an ordinary differential equation solver, and the function modFit acted as a wrapper which takes the residuals as input and adjusts parameters based 
Table 2: Thrombopoiesis baseline model parameter values.

\begin{tabular}{cc}
\hline Parameter & Value \\
\hline$\alpha$ & $2.4 \mathrm{~d}^{-1}$ \\
$\gamma$ & $1.4 \mathrm{~d}^{-1}$ \\
$\delta$ & $0.2 \mathrm{~d}^{-1}$ \\
$\psi_{0}$ & $0.11 \mathrm{~d}^{-1}$ \\
$n$ & 4 \\
$\lambda$ & 0.25 \\
$\sigma$ & 3000 \\
$\theta_{1}$ & 1 \\
$\theta_{2}$ & 0.1 \\
$\theta_{3}$ & 0.0001 \\
\hline
\end{tabular}

on the specified optimization algorithm, which in this instance was the Levenberg-Marquardt algorithm.

To calculate parameter confidence intervals, the Markov Chain Monte Carlo (MCMC) method with the adaptive Metropolis algorithm was used [18,9]. Given an initial set of parameters, an estimate for the parameter covariance matrix, and a function which returns model output residuals, this algorithm randomly steps through the parameter space, selecting new potential parameter combinations. The likelihood of the new parameter combination is determined, and if it is greater than the current state, the new parameter set is chosen. If the likelihood of the new parameter combination is less than the current state, the algorithm selects the new state with probability $\alpha$, where $\alpha$ equals the probability of the new state divided by the probability of the current state. This algorithm was performed in $\mathrm{R}$, using modMCMC which is also a function of the FME library.

Data from a retroactive study on burn patients was used for optimization [21]. This study was selected based on the study size, early initial platelet level recordings, prolonged duration, and detailed information for three separate groups in which subjects were grouped according to burn size. The initial platelet count was used as the baseline level when optimizing. If significant decreases in platelet count occurred before the first recorded value, the optimized model prediction would underestimate platelet counts, however all three groups had average initial platelet counts within the normal range for humans $\left(140-440 \times 10^{3} / \mu L ;[34]\right)$. Data from later time points come from fewer patients and are coupled with the existence of complications and/or multiple surgeries that warranted a longer hospital stay. For this reason, not as much emphasis was placed on matching the data from these time points. The study divided the patients into three groups based on the fraction of surface area that was burned $(S)$. The first group contained subjects where $0.015 \leq S<0.15$, the second contained subjects where $0.15 \leq S<0.30$, and the third contained subjects where $0.30 \leq S \leq 0.90$. For each group, the data provided on platelet counts is the average value within each group at each specified time. When optimizing and comparing the model output to the data, the input to the model was the midpoint within each range.

\section{Results and Discussion}

A mathematical model of thrombopoiesis that accurately depicts the observed effects of burn on blood platelet levels has been developed. These effects include (1) an initial burnsize dependent decrease in thrombocyte levels followed by (2) a sustained thrombocytosis. The results of the optimization and subsequent validation and simulations are presented and discussed below. 
Table 3: Optimized parameter values for the human thrombopoiesis model. The parameter values represent the set of parameters that minimized the residual sum-of-squares following the optimization and MCMC analysis. The $95 \%$ confidence interval represents the interval that $95 \%$ of the accepted parameter sets fell into during the MCMC analysis. The bounds column represents the boundary constraints placed on the parameters during the optimization and MCMC analysis.

\begin{tabular}{lccc}
\hline Parameter & Value & $95 \%$ Confidence Interval & Bounds [min, max] \\
\hline$a_{0}\left(\mathrm{~d}^{-1}\right)$ & 0.02 & $0.019-0.066$ & {$[0,0.2]$} \\
$b_{0}$ & 1.70 & $1.83-3.52$ & {$[0,20]$} \\
$c_{0}$ & 10 & $5.51-9.83$ & {$[1,10]$} \\
\hline
\end{tabular}

\subsection{Optimization}

The optimization results for the human model are presented in Figure 2. Three different simulations overlaid on the corresponding optimization data are presented, demonstrating that at lower \%TBSA the initial decrease in platelet count is less drastic. The optimized simulation captures the burn-size dependent nadir as well as the following entry into a state of thrombocytosis. The resulting optimized parameter values and confidence intervals are given in Table 3 . For $b_{0}$, the optimized value does not fall within the given confidence interval. This means that although the optimized value represents the value which minimizes the sum of squares, there are not many neighboring values that give as good of fits as there are within the given $95 \%$ confidence interval. These parameter values imply that following a $20 \%$ burn, the rate of production of the generic mediator increases by $1.70 \cdot 0.2^{1 / 10} \cdot 100 \%$ or $145 \%$, and 50 days are required to reduce this change by half.

Optimizing model output to human data is challenging due to potential confounding factors including treatment, development of sepsis, and surgery. Although the initial decline in platelets is thought to be due to consumption of platelets through coagulation, there are a variety of other potential causes including dilution due to the administration of resuscitation fluids, bone-marrow depression, and drug-induced thrombocytopenia. Drugs known to cause thrombocytopenia such as silver sulphadiazine, heparin, morphine and paracetamol are commonly used when treating patients with burn. The thrombocytosis period could also be artificially caused by the administration of transfusions, but typically transfusions are only advised when the nadir is below $50 \times 10^{9}$ platelets which was not observed in the main study used for optimization [21].

\subsection{Validation results}

\subsubsection{Optimized parameter values}

Circulating levels of TPO increase in burn patients (15-40\% TBSA) without sepsis by $123 \pm 48 \%$ (using only the error on the TPO measurement; [19]). Patients with low platelet levels $\left(<100 \cdot 10^{9} \mathrm{~L}^{-1}\right)$ were excluded from this study, so the increased TPO levels were caused by more than just the thrombopenic state. If the TPO values were measured approximately 1 day postburn, according to our model we would expect to see a $167 \%$ increase in TPO levels. This calculation assumes (1) the postburn measurement time is 1 day and (2) alterations in TPO levels are the only contribution to the increased generic mediator production. This prediction falls within the error range of the observed change in TPO levels $(123 \pm 48 \%)$.

Serial data is available on IL-6 concentrations in humans for up to 65 days postburn [23]. Since IL-6 is known to lead to increases in circulating TPO levels [14], we can use this data to validate the recovery time (the value of $a_{0}$ ) obtained in the optimization. The recovery function $B /\left(1+a_{o} t\right)$ was fit to data on IL-6 levels and a value of $0.04 \mathrm{~d}^{-1}$ with a standard error of $0.01 \mathrm{~d}^{-1}$ was obtained for $a_{0}$ (data not shown). This value is twice that which was 


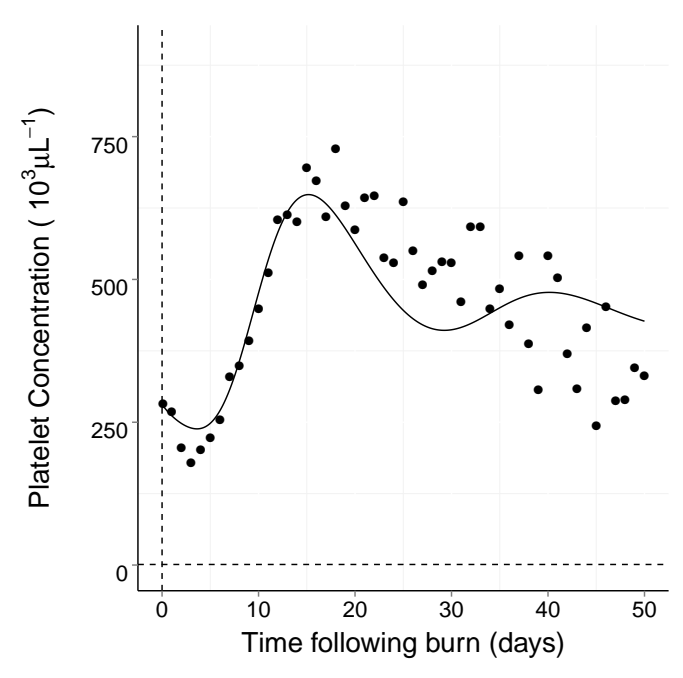

iA.

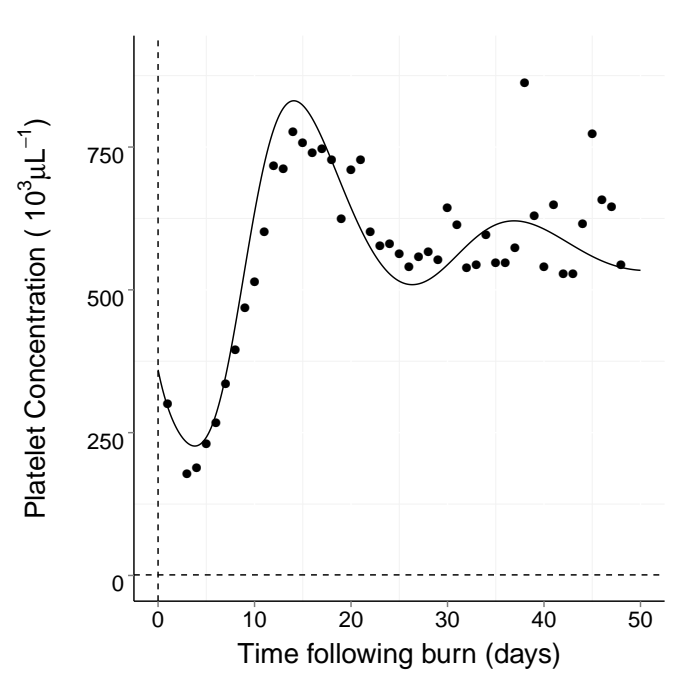

SA.

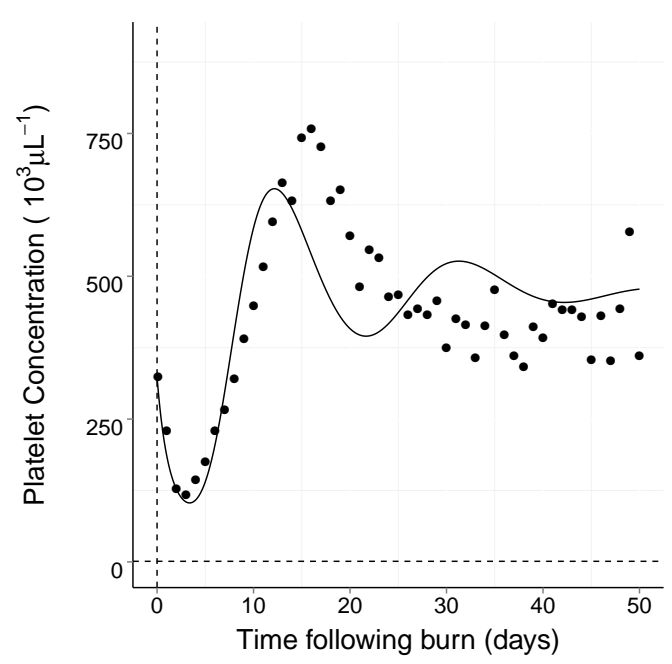

Figure 2: Optimized simulation results overlaid on data used for optimization. In the subfigure legend 'simulation' refers to the input value of $S$ for the presented model (black line) and 'data' represents the range of burn sizes for the presented data (black dots, data given is mean value). Data digitized from [21]. 
obtained during the optimization suggesting the system might recover at a faster rate than our model predicts. However, this experimental result does fall within our calculated $95 \%$ confidence interval.

\subsubsection{Model output}

The human thrombopoiesis model was validated against data from studies on platelet counts following burn in humans. These studies provide information on the mean platelet count from subjects with burn sizes in a specified range. Validating the model against this data is challenging because (1) data are given for a range of burn sizes without information on the mean burn size, (2) no baseline value is provided and often early blood cell count information is not available, and (3) these subjects have received various treatments which likely affect the observed platelet counts.

The validation was performed by initiating the model at the mean normal thrombocyte concentration observed in humans, $250 \cdot 10^{6} \mu L^{-1}$ [34], and two model outputs were calculated; one based on the lower end of the range for \%TBSA of subjects within the group and one at the higher end of the range for \%TBSA. The results of these comparisons are shown in Figure 3. Visual inspection reveals that the model is able to consistently match the trends observed.

\subsection{Simulation results}

Figure 4 gives the simulation results showing how increasing burn size affects the concentration of cells in each compartment. In the $X_{1}$ compartment there is an immediate increase in cell concentration, while in the $X_{2}$ compartment, this increase is slightly delayed. Even at burn sizes of $10 \%$, the increased progenitor cell concentrations are still very large. This is due to the large value of $c_{0}$ which causes small burns to still lead to large effects in mediator production changes. For the $X_{3}$ compartment, or platelets in the blood stream, there is the expected initial decrease in platelet count. At lower burn sizes (10\%) this decrease is much less drastic due to the linear relationship between burn size and magnitude of the platelet decay rate change. The resulting thrombocytosis is actually larger in subjects with smaller burns. This is due to the increased rate of platelet decay.

Simulations show the model accurately reflects two trends: (1) the platelet nadir decreases with larger burn wound size and (2) the magnitude of the subsequent thrombocytosis is not positively correlated with burn wound size. However, further investigation would be required to determine whether a negative correlation between burn size and extent of thrombocytosis exists. Its important to note that these trends apply to burn wound sizes between $10 \%$ and $100 \%$. At very small \%TBSA, our model predicts a positive correlation between burn size and the thrombocytosis magnitude. This is a realistic prediction; however, due to a lack of data from burn patients with $<10 \%$ TBSA, model outputs should be used with caution. The model may predict too large of a thrombocytosis following small burns.

\section{Conclusion}

We have presented a model of thrombopoiesis incorporating the effects of burn. This model includes descriptions of key physiological processes, and simulations using this model produce outputs that reflect key trends in thrombocyte kinetics following burn. However, several of our simplifications and assumptions warrant further investigation. For example, although we model platelet lifespan with an exponential decay function, some data from radiotracer studies support a linear distribution in platelet lifespans [6]. Other models of platelet decay, for example, a model incorporating both a homeostatic loss and a senescent loss could also be investigated [11]. Secondly, our model assumes that the maximum effect of burn occurs immediately following injury and gradually returns to normal. However, 

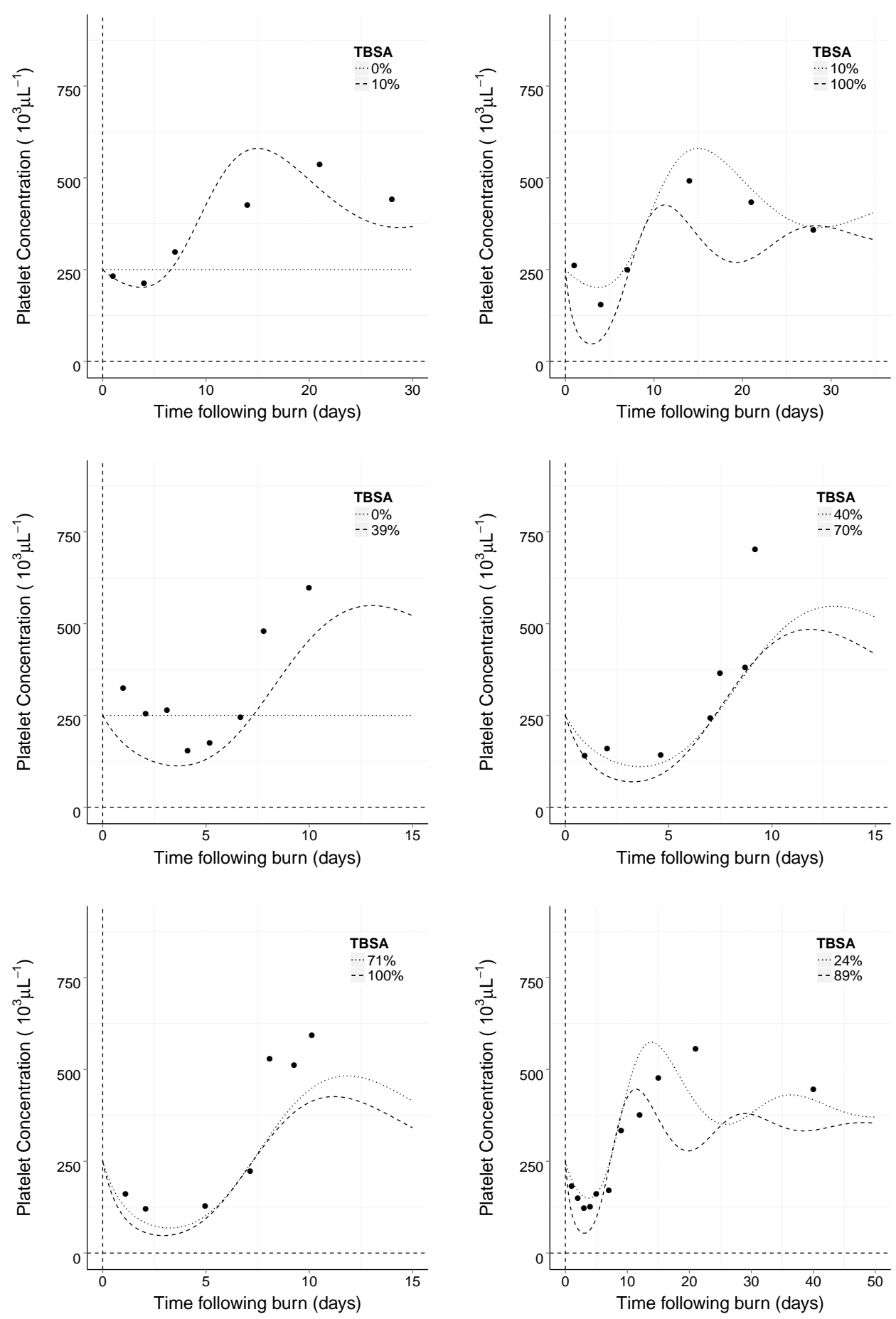

Figure 3: Comparison of thrombopoiesis model simulations overlaid on data reserved for validation. Data points represent mean values for subjects reported with \%TBSA within the given range. Simulations represent upper and lower bounds of the \%TBSA ranges. Data were obtained from [24] for $(\mathrm{a}, \mathrm{b}),[3]$ for $(\mathrm{c}, \mathrm{d}, \mathrm{e})$, and [29] for (f). 

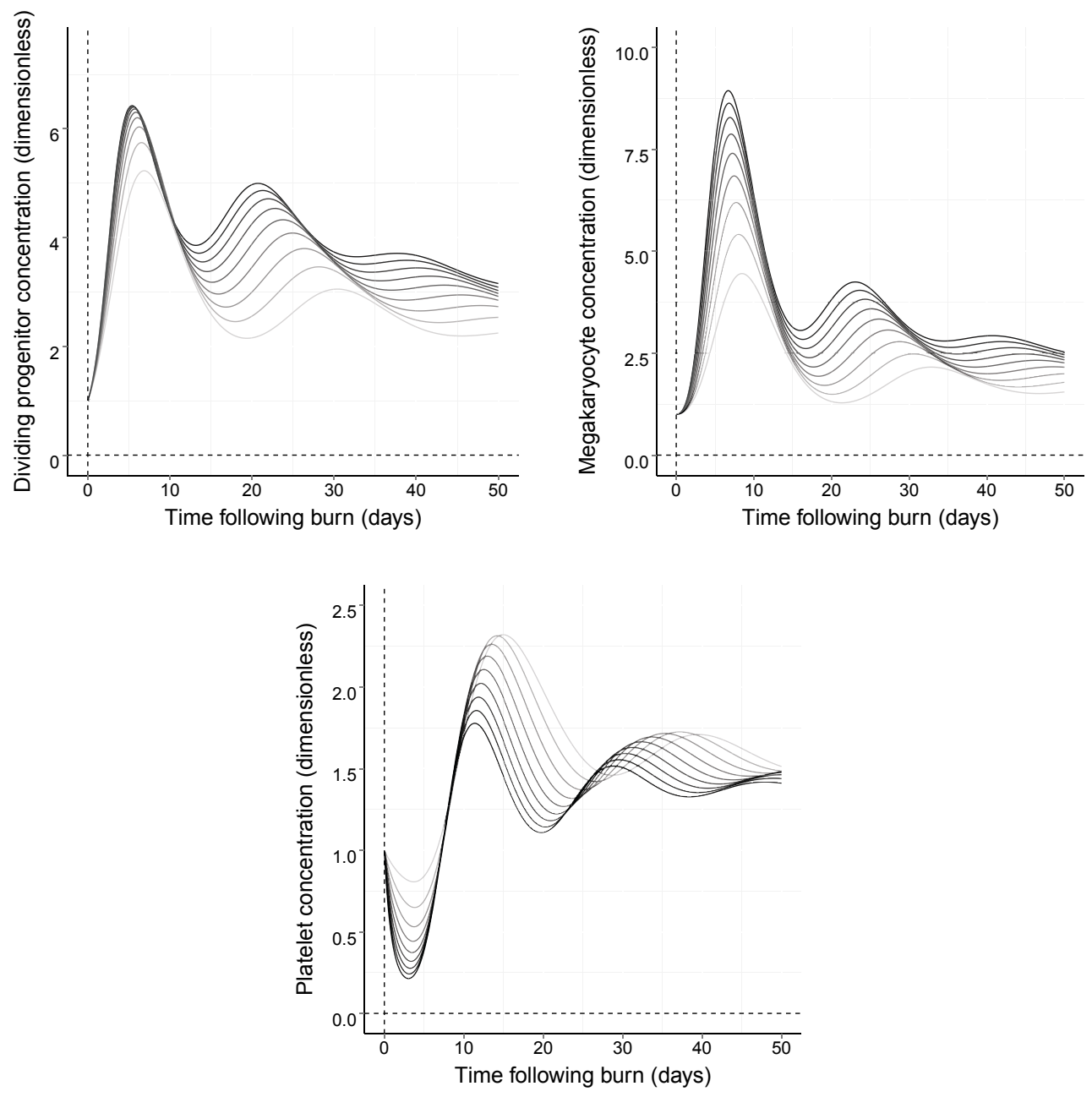

Figure 4: Model simulations performed for various burn sizes. Output given for 10$100 \%$ TBSA in $10 \%$ increments. Darker lines represent larger burn sizes. Graphs shown for the $X_{1}$ compartment (top left), the $X_{2}$ compartment (top right), and the $X_{3}$ compartment (bottom). The $X_{2}$ compartment represents the normalized sum of all the $X_{2}$ subcompartments.

since mediator release can impact hematopoiesis, it likely takes some time before thermal injury has a maximal impact on thrombopoietic rates. Studies showing peak IL-6 levels occur three to six days after burn support this hypothesis [8, 37]. Also, burn size may affect the time it takes for kinetic rates to return to normal rather than the magnitude of kinetic rate alterations [23]. However, additional experimental data will be needed in order to better understand these topics and to further refine the thrombopoiesis model for burn. Investigation of these points affords an opportunity for experimentalists and modelers to collaborate to further our understanding of the underlying mechanisms involved in thermal injury.

Our future goals in model development will be to merge the effects of radiation with those of burn on thrombopoiesis. Evaluation of the merged models will also help test the premises from which the model was constructed. If the combined models provide rational outputs, we can then use the model to provide insight on the mechanisms behind mortality due to combined injury and to predict the time course of injury and recovery. 


\section{References}

[1] American Burn Association, 2012. Burn Incidence and Treatment in the United States: 2012 Fact Sheet.

[2] S. Asano, A. Okano, K. Ozawa, T. Nakahata, T. Ishibashi, K. Koike, H. Kimura, Y. Tanioka, A. Shibuya, T. Hirano, 1990. In vivo effects of recombinant human interleukin-6 in primates: stimulated production of platelets. Blood, 75.8, 1602-1605.

[3] C. R. Baxter, 1974. Fluid volume and electrolyte changes of the early postburn period. Clinics in Plastic Surgery, 1.4, 693-709.

[4] H. Carsin, M. Assicot, F. Feger, O. Roy, I. Pennacino, H. Le Bever, P. Ainaud, C. Bohuon, 1997. Evolution and significance of circulating procalcitonin levels compared with IL-6, TNF alpha and endotoxin levels early after thermal injury. Burns, 23.3, $218-224$.

[5] A. Drost, D. Burleson, W. G. Cioffi, B. S. Jordan, A. D. Mason, B. A. Pruitt, 1993. Plasma cytokines following thermal injury and their relationship with patient mortality, burn size, and time postburn. The Journal of Trauma-Injury, Infection, and Critical Care, 35.3, 335-339.

[6] K. Eurenius, R. F. Mortensen, P. M. Meserol, P. W. Curreri, 1972. Platelet and megakaryocyte kinetics following thermal injury. Journal of Laboratory and Clinical Medicine, 79.2, 247-257.

[7] D. H. Graessle, 2000. Simulation of Radiation Effects Using Biomathematical Models of the Megakaryocytic Cell Renewal System. Universität Ulm, Medizinische Fakultät.

[8] Y. Guo, C. Dickerson, F. J. Chrest, W. H. Adler, A. M. Munster, R. A. Winchurch, 1990. Increased levels of circulating interleukin 6 in burn patients. Clinical Immunology and Immunopathology, 54.3, 361-371.

[9] H. Haario, E. Saksman, J. Tamminen, 2001. An adaptive Metropolis algorithm. Bernoulli, 7.2, 223-242.

[10] L. A. Harker, L. K. Roskos, U. M. Marzec, R. A. Carter, J. K. Cherry, B. Sundell, E. N. Cheung, D. Terry, W. Sheridan, 2000. Effects of megakaryocyte growth and development factor on platelet production, platelet life span, and platelet function in healthy human volunteers. Blood, 95.8, 2514-2522.

[11] J. K. Hersh, E. G. Hom, M. E. Brecher, 1998. Mathematical modeling of platelet survival with implications for optimal transfusion practice in the chronically platelet transfusion-dependent patient. Transfusion, 38.7, 637-644.

[12] T. A. Housinger, C. Brinkerhoff, G. D. Warden, 1993. The relationship between platelet count, sepsis, and survival in pediatric burn patients. Archives of Surgery, 128.1, 6567.

[13] T. Ishibashi, H. Kimura, Y. Shikama, T. Uchida, S. Kariyone, T. Hirano, T. Kishimoto, F. Takatsuki, Y. Akiyama, 1989. Interleukin-6 is a potent thrombopoietic factor in vivo in mice. Blood, 74.4, 1241-1244.

[14] A. Kaser, G. Brandacher, W. Steurer, S. Kaser, F. A. Offner, H. Zoller, I. Theurl, W. Widder, C. Molnar, O. Ludwiczek, M. B. Atkins, J. W. Mier, H. Tilg, 2001. Interleukin- 6 stimulates thrombopoiesis through thrombopoietin: role in inflammatory thrombocytosis. Blood, 98.9, 2720-2725.

[15] K. Kaushansky, 2005. The molecular mechanisms that control thrombopoiesis. Journal of Clinical Investigation, 115.12, 3339-3347.

[16] E. E. Kovalev, O. A. Smirnova, 1996. Estimation of radiation risk based on the concept of individual variablity of radiosensitivity. AFRRI-CR96-1, Bethesda, MD: Armed Forces Radiobiology Research Institute.

[17] A. Kowal-Vern, J. M. Walenga, D. Hoppensteadt, M. Sharp-Pucci, R. L. Gamelli, 1994. Interleukin-2 and interleukin- 6 in relation to burn wound size in the acute phase of thermal injury. Journal of the American College of Surgeons, 178.4, 357-362.

[18] M. Laine, 2008. Adaptive MCMC Methods with Applications in Environmental and Geophysical Models. Finish Meteorological Institute. 
[19] E. Lupia, O. Bosco, F. Mariano, A. E. Dondi, A. Goffi, T. Spatola, A. Cuccurullo, P. Tizzani, G. Brondino, M. Stella, G. Montrucchio, 2009. Elevated thrombopoietin in plasma of burned patients without and with sepsis enhances platelet activation. Journal of Thrombosis and Haemostasis, 7.6, 1000-1008.

[20] J.L.S. Macedo, J.B. Santos, 2007. Predictive factors of mortality in burn patients. Revista do Instituto de Medicina Tropical de São Paulo, 49.6, 365-370.

[21] R. E. Marck, H. L. Montagne, W. E. Tuinebreijer, R. S. Breederveld, 2013. Time course of thrombocytes in burn patients and its predictive value for outcome. Burns, 39.4, $714-722$.

[22] M. Mirsaeidi, P. Peyrani, S. Aliberti, G. Filardo, J. Bordon, F. Blasi, J. A. Ramirez, 2010. Thrombocytopenia and thrombocytosis at time of hospitalization predict mortality in patients with community-acquired pneumonia. CHEST Journal, 137.2, 416420.

[23] M. W. Nijsten, C. E. Hack, M. Helle, H. J. Ten Duis, H. J. Klasen, L. A. Aarden, 1991. Interleukin-6 and its relation to the humoral immune response and clinical parameters in burned patients. Surgery, 109.6, 761-767.

[24] M. Pavić, L. Milevoj, 2007. Platelet count monitoring in burn patients. Biochemia Medica, 17.2, 212-219.

[25] T. C. Pellmar, G. D. Ledney, 2005. Combined injury: radiation in combination with trauma, infectious disease, or chemical exposures. Bethesda, MD: Armed Forces Radiobiology Research Institute.

[26] R Core Team, 2013. R: A language and environment for statistical computing. R Foundation for Statistical Computing. Vienna, Austria.

[27] B. Schluter, B. Konig, U. Bergmann, F. E. Muller, W. Konig, 1991. Interleukin 6-A potential mediator of lethal sepsis after major thermal trauma: Evidence for increased IL-6 production by peripheral blood mononuclear cells. The Journal of Trauma-Injury, Infection, and Critical Care, 31.12, 1663-1670.

[28] M. Scholz, A. Gross, M. Loeffler, 2010. A biomathematical model of human thrombopoiesis under chemotherapy. Journal of Theoretical Biology, 264.2, 287-300.

[29] T. Simon, P. Curreri, L. Harker, 1977. Kinetic characterization of hemostasis in thermal injury. The Journal of Laboratory and Clinical Medicine, 89.4, 702-711.

[30] K. Skomorovski, H. Harpak, A. Ianovski, M. Vardi, T. P. Visser, S. C. C. Hartong, H. H. D. M. Vliet, G. Wagemaker, Z. Agur, 2003. New TPO treatment schedules of increased safety and efficacy: Pre-clinical validation of a thrombopoiesis simulation model. British Journal of Haematology, 123.4, 683-691.

[31] O. A. Smirnova, 2011. Environmental Radiation Effects on Mammals. Springer.

[32] O. A. Smirnova, 2012. Comparative analysis of the dynamics of thrombocytopoietic, granulocytopoietic, and erythropoietic systems in irradiated humans: a modeling approach. Health Physics, 103.6, 787-801.

[33] K. Soetaert, T. Petzoldt, 2010. Inverse modelling, sensitivity and Monte Carlo analysis in R using package FME. Journal of Statistical Software, 33.3, 1-28.

[34] J. Valentin, 2002. Basic anatomical and physiological data for use in radiological protection: Reference values. ICRP Publication 89. Annals of ICRP, 32.3-4, 1-277.

[35] Y. U. Wang, H. T. Tang, S. H. Xia, S. H. Zhu, B. Ma, W. Wei, Y. Sun, K. Y. Lv, 2010. Factors affecting survival in adult patients with massive burns. Burns, 36.1, 57-64.

[36] S. Wells, M. Sissons, P. S. Hasleton, 1984. Quantitation of pulmonary megakaryocytes and fibrin thrombi in patients dying from burns. Histopathology, 8.3, 517-527.

[37] F. L. Yeh, W. L. Lin, H.D. Shen, R. H. Fang, 1999. Changes in circulating levels of interleukin 6 in burned patients. Burns, 25.2, 131-136. 